livraisons

d'Histoire

de l'Architecture

\section{Livraisons de l'histoire de l'architecture}

$23 \mid 2012$

Varia I

\title{
Le choix de l'unique. Un aperçu des politiques de sélection lors des ventes du mobilier des résidences royales
}

Detecting what's unique: an insight into choice's policy before roy al furniture auctions during French revolution

Die Entscheidung für Einzelstücke. Einblick in die Auswahlmethoden bei den staatlichen Verkäufen des Mobiliars der königlichen Wohnstätten

\section{Rémi Gaillard}

\section{(2) OpenEdition}

\section{Journals}

Édition électronique

URL : http://journals.openedition.org//ha/121

DOI : 10.4000//ha. 121

ISSN : 1960-5994

Éditeur

Association Livraisons d'histoire de l'architecture - LHA

Édition imprimée

Date de publication : 15 juin 2012

ISSN : 1627-4970

\section{Référence électronique}

Rémi Gaillard, «Le choix de l'unique. Un aperçu des politiques de sélection lors des ventes du mobilier des résidences royales », Livraisons de l'histoire de l'architecture [En ligne], 23 | 2012, mis en ligne le 05 juin 2013, consulté le 02 mai 2019. URL : http://journals.openedition.org/lha/121 ; DOI : 10.4000/

Iha. 121

Ce document a été généré automatiquement le 2 mai 2019.

Tous droits réservés à l'Association LHA 


\title{
Le choix de l'unique. Un aperçu des politiques de sélection lors des ventes du mobilier des résidences royales
}

\author{
Detecting what's unique: an insight into choice's policy before royal furniture \\ auctions during French revolution \\ Die Entscheidung für Einzelstücke. Einblick in die Auswahlmethoden bei den \\ staatlichen Verkäufen des Mobiliars der königlichen Wohnstätten
}

Rémi Gaillard

Le 28 frimaire de l'an II, le député Mathieu, ancien avocat, monta à la tribune de la Convention. Il venait réclamer une tête : celle de la commission des monuments. Cette institution était née de l'agrégation de plusieurs petites commissions, crées à partir d'initiatives diverses dans la suite de la nationalisation des biens ecclésiastiques. Elle s'était constituée en «Commission conservatrice des monuments » au printemps 1792 et avait reçu son organisation définitive à l'automne de la même année. L'Exposé succinct qu'elle fit publier juste avant les attaques de Mathieu résume parfaitement ses missions : «sauver des mains de l'ignorance et la malignité les monuments des arts, et [...] en prévenir les ventes inconsidérées qui en auraient dépouillé la Nation ${ }^{1}$. » Ce 28 frimaire, Mathieu lit son rapport de quatre pages au terme duquel il demande la suppression de la Commission des monuments et son remplacement par la commission temporaire des arts, dont il saluait le civisme, le zèle et l'utilité. Au cœur de la démonstration, Mathieu dévoilait son principal argument en accusant la Commission de négligence manifeste dans sa politique de distraction des objets pouvant « servir à l'instruction et aux arts » et posa la question: «Dans ceux qui s'offraient à elle, a-t-elle fait un bon choix ${ }^{2}$ ?» La réponse fut bien entendu négative et pour convaincre son auditoire, Mathieu présenta son l'exemple de Versailles, après avoir évoqué la dilapidation préjudiciable de colonnes de marbre tirées de la ci-devant abbaye de Franciade et des collections de l'émigré Choiseul-Gouffier. 
L'on a vendu, environ 8000 livres, quatre tables de bois pétrifié qui avaient appartenu à la femme du tyran et qui, estimées à leur valeur, ne pouvaient l'être à une somme moindre de 150000 livres, à raison de leurs dimensions peu communes et du travail d'art par lequel on a embelli ce rare produit de la nature [...]. Déjà un jardinier anglais, établi à Sèvres, était en marché et se proposait d'en faire l'emplette, non pour lui-même, comme on peut le croire, mais indubitablement pour ses concitoyens de la Grande-Bretagne ${ }^{3}$.

2 À la grossièreté de l'erreur d'appréciation s'ajoutait donc, dans l'argumentation de Mathieu, la possibilité que cette dernière bénéficiât aux ennemis de la République. L'exemple est fondamental, car il pose en des termes simples le cadre et les contradictions des politiques de tri et de sélection révolutionnaires à l'épreuve de l'immense quantité d'objets de tout type et de toute valeur mis en vente au profit de la Nation. La critique de Mathieu est en fait double et presque contradictoire : non seulement il accuse la Commission d'avoir fait vendre à vil prix les tables (dilapidation), mais regrette encore qu'elles n'aient pas échappé à l'aliénation - ce que le « travail d'art » avec lequel elles avaient été réalisées, pourtant, justifiait. On touche ainsi du doigt les deux grands types de sélection qu'opérèrent, sur le terrain, les révolutionnaires, qu'ils soient représentants du pouvoir ou membres des commissions artistiques. Bien loin de cantonner la sélection à la distraction de « monuments » que l'on souhaitait, par mise à la disposition du Muséum, rendre inaliénables, les commissaires ont également mis de côté des pièces dont la vente, pour des raisons typologiques ou proprement « marchandes » devaient être momentanément conservées pour être ensuite aliénées, mais selon d'autres modalités. Enfin, l' « affaire des tables pétrifiées » pose une dernière question, essentielle dans l'étude des processus de sélection à l'œuvre lors des ventes du mobilier royal, celle de la place du mobilier, entendu dans son sens restreint de "meubles meublants » (tapisseries, vases, meubles d'ébénisterie, lustres et girandoles, etc.), dans les sélections «patrimoniales » opérées par les commissions artistiques ${ }^{4}$.

3 Les législateurs révolutionnaires n'ont produit aucun texte général donnant le cadre précis des distractions qu'il fallait opérer préalablement à la vente, et c'est ce qui explique en partie leur décision de confier cette tâche à des commissions artistiques particulières. Ces dernières avaient non seulement la responsabilité, sur le terrain, du " choix », mais étaient également chargées, en cultivant une certaine expertise, de mener une réflexion sur les lois qui encadreraient les politiques de sélection patrimoniale. L'acte de naissance de la commission des monuments, le décret du 18 octobre 1792, la chargeait « de prendre connaissance des monuments qui doivent être conservés pour la gloire des arts et des sciences [...], de veiller à leur conservation » et, en définissant ses compétences en matière de tri, lui demandait enfin, en concertation avec les comités, de présenter à la Convention les projets de décrets «relatifs à la distraction" de ces monuments du nombre des effets mobiliers devant être vendus ${ }^{5}$. Elle ne produisit en fait que très peu de textes législatifs, agissant surtout par la rédaction d'une importante quantité de mémoires et d'instructions destinés à guider les administrations locales dans les opérations de tri auxquelles la Commission ne pourrait pas participer elle-même - à savoir la majorité. À la lecture de ces documents, une première observation peut être formulée : la distraction patrimoniale n'est presque envisagée que pour les productions des beaux-arts. Outre les monuments dont l'ancienneté légitime la sélection («les monuments de l'antiquité et du Moyen Âge »), ce sont les «statues, tableaux, dessins et autres objets relatifs aux beaux-arts » qui doivent faire l'objet de la majorité des mesures conservatoires $^{6}$. Une des expressions employées dans le procès-verbal de la première 
inspection de la commission des monuments, à la fin de l'été 1793, à la résidence de Marly, est à cet égard significative : les commissaires annoncent aux organisateurs de la vente qu'ils viennent choisir «les objets de peinture et sculpture» susceptibles d'échapper à la dispersion. Cet intitulé sanctionne l'exclusion d'office de cette sélection des pièces mobilières les plus précieuses, tout en faisant des œuvres d'art, peintes et sculptées, l'unique champ d'action possible du choix patrimonial révolutionnaire. Il ne faut cependant pas se fier à cette simple distinction typologique: le mobilier aussi fait l'objet des attentions des membres des commissions artistiques. Ils le confirment dans une Instruction pour les commissaires aux ventes, rédigée au début de l'année 1793 et dans laquelle ils rappellent l'étendue possible des distractions dont ils ont la responsabilité: «Plusieurs des commissaires aux ventes ne paraissent pas assez convaincus de l'étendue des pouvoirs des membres de la Commission [des monuments], tous ne savent pas que l'exercice de ces pouvoirs ne doit point se borner à l'inspection des tableaux, gravures, médailles, bibliothèques [...], mais qu'il comprend aussi l'examen des [...] tapisseries et autres meubles meublants qui peuvent présenter quelque singularité remarquable ou un travail précieux et instructif pour le progrès de l'art ${ }^{7}$. » Cet élargissement du champ de sélection fut enfin doublé de l'élaboration de mesures conservatoires provisoires. En effet, la Révolution ne s'est pas simplement contentée de séparer le bon grain de l'ivraie en se limitant seulement à choisir entre l'aliénation et la conservation. Par suite de l'adoption, le 27 juillet 1792, du principe de la vente des biens des émigrés, de nombreuses réflexions sont menées pour l'élaboration d'une troisième voie intermédiaire, passant également par la sélection et la mise en réserve. Le ministre de l'Intérieur de l'époque, Roland de la Platière, à qui est confié le soin d'élaborer les détails des processus d'aliénation, joue un rôle important dans cette réflexion. Dans une lettre-circulaire qu'il adresse le 5 novembre 1792 à l'administration des départements de Paris, de l'Oise et de la Seine-et-Oise, il demande que soient réservés les objets d'un certain prix dont l'aliénation devrait faire l'objet de procédures spécifiques et différenciées. Il l'explicite dans une note qu'il joint à sa circulaire, intitulée "La conservation des objets d'art ", note assez virulente à l'égard des administrations domaniales et dans laquelle il propose :

Que beaucoup de choses, plus précieuses par l'art que par la matière, et que cependant l'on jugerait ne devoir pas conserver, parce que elles dépendraient plus encore du caprice que du goût, il est intéressant de n'en pas précipiter la vente et de les réserver dans des dépôts jusqu'à des moments plus favorables ${ }^{8}$.

Une fois ces deux possibilités de sélection offertes, encore fallait-il les mettre en application. On le constate aisément, la principale difficulté venait de l'absence de critères objectifs permettant de mener les distractions avec constance. Dans son entreprise toute entière dévouée à la cause de son héros Alexandre Lenoir, Louis Courajod a particulièrement insisté sur cette subjectivité dommageable, mais inhérente à la politique de sélection. "Les œuvres d'art avaient leur tribunal révolutionnaire ${ }^{9}$ ", et les tournées d'inspection des membres des commissions artistiques, parisienne et départementale, faisaient office de procès. Malgré les fluctuations du choix et le silence de la majorité des documents, quelques pistes peuvent être évoquées afin d'éclairer les motifs de la sélection.

Dans la masse du mobilier des résidences royales, une première catégorie de meubles est choisie en fonction de son utilité ; il ne s'agit pas à proprement parler d'une sélection patrimoniale, mais l'objet échappe à l'aliénation pour servir de support, de présentoir ou de décor, inséré et intégré dans l'espace muséographique naissant. Il est donc choisi sur critère fonctionnel. Ainsi lors de sa seconde visite à Marly, la commission des monuments 
représentée par le sculpteur Louis Boizot, sélectionne des banquettes et des tabourets couverts de Savonnerie ; ils lui paraissent alors «utiles à placer dans la longueur du Muséum $^{10}$ ». En visite au château de Compiègne à la fin du mois de novembre 1792, des membres de la même commission insèrent dans l'inventaire des objets à distraire des ventes des pliants peints et des portières "dans le même genre ", car «si dans le milieu du Muséum on faisait une espèce de tribune ou de centre de repos, il conviendrait de placer autour ces meubles ${ }^{11} »$. Ailleurs, des tables et consoles sont sélectionnées pour le même motif ; on peut en apercevoir dans la Grande Galerie peinte vers 1800 par Hubert Robert (ill.1).

\section{1: Hubert Robert, La Grande Galerie du Louvre entre 1801 et 1805}

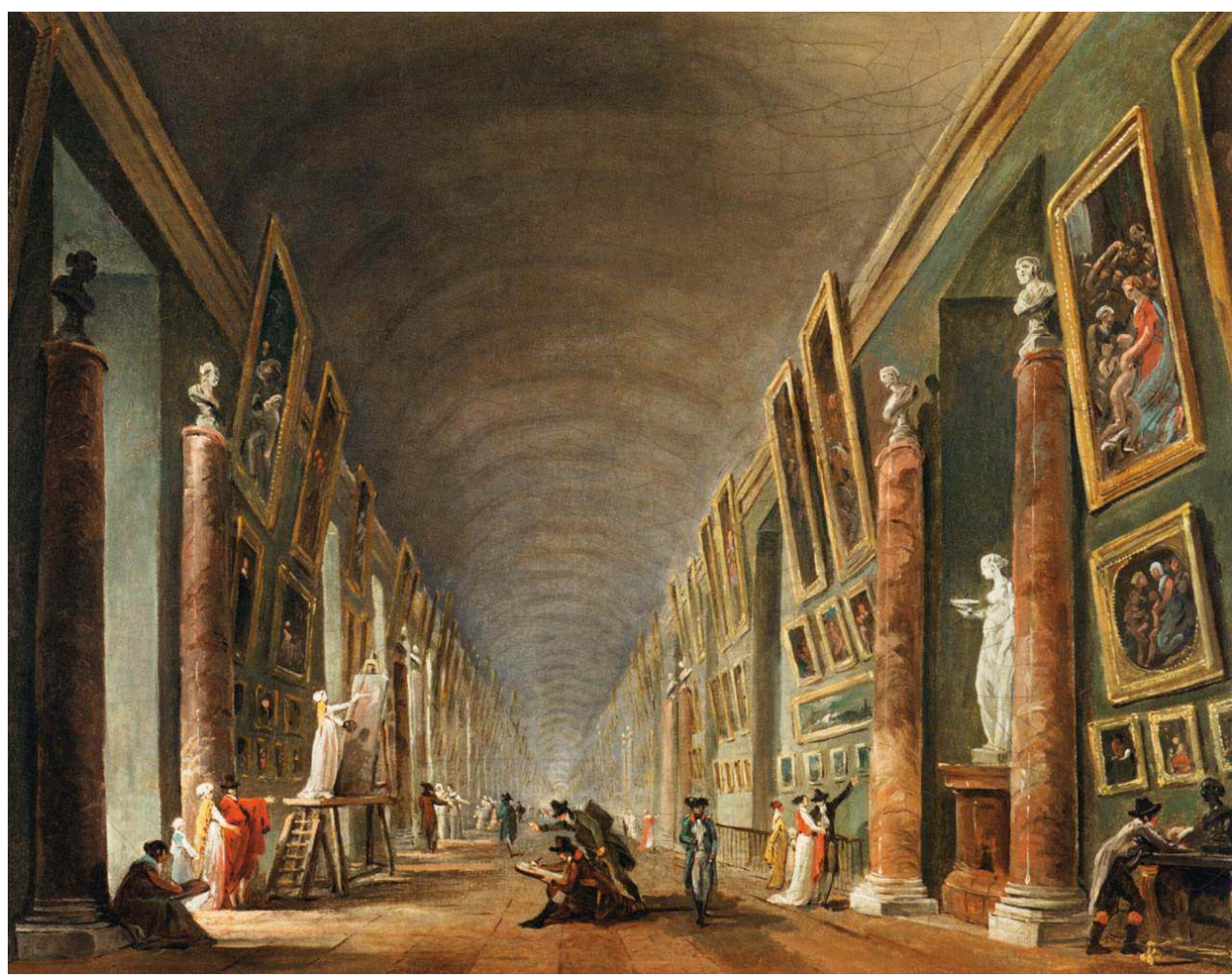

Sans date, musée du Louvre.

Cl. Musée du Louvre/A. Dequier - M. Bard.

Viennent ensuite les pièces mobilières que l'on juge "dignes d'être conservées ", non pour l'ameublement des salles du Louvre, mais pour rejoindre les collections du musée. Même peu nombreuses, ces sélections présentent quelques points communs. La préciosité du matériau peut apparaître comme le premier motif de distraction: la table et la console à dessus de pierre dure, son piétement en bois sculpté doré ou peint, sont les premiers à tirer leur épingle du jeu de la sélection. À Compiègne notamment, une table de porphyre est ainsi désignée ${ }^{12}$; le commissaire-artiste Boizot retire des appartements de Marly une paire de « tables de marbre de rapport de Florence, cernées de bronze, et leurs pieds de bois doré ", mais aussi des tables d'albâtre tirées des vestibules, sur piétement de bois peint. De même, s'il sélectionne une petite commode à la régence en bois de rapport, c'est, dit-il, «à cause de son marbre $»^{13}$. Un autre critère fait régulièrement son apparition, et entraîne constamment la distraction, c'est l'attribution à l'ébéniste Boulle. Marianne Clerc a mis en lumière l'estime accordée à cet artisan dont la marqueterie 
caractéristique fait, auprès des commissaires, unanimement mouche. Ayant analysé les objets d'art saisis chez les émigrés et les condamnés à partir des registres du dépôt national de la rue de Beaune où ils étaient entreposés, elle révèle que plus de $40 \%$ des meubles retenus par les commissions sont attribués au maître ébéniste..$^{14} \mathrm{Il}$ ne faut donc pas s'étonner de voir mis en réserve par le graveur Moreau et le peintre Lemonnier à la chancellerie de Compiègne un couple de «belles commodes à tombeau, du très beau Boule [sic] ». Boizot lui aussi retient du mobilier de Marly une commode "à trois tiroirs en bois de placage [...], assez précieuse par son travail entier, estimé de Boulle $»^{15}$. Ces catégories de meubles posent rarement problème. Ils sont sélectionnés sans hésitation, sur des critères que l'on peut considérer - puisqu'ils sont successivement appliqués en plusieurs endroits - comme communément admis. D'autres objets suscitent, dans le cours $\mathrm{du}$ processus de sélection, questionnements et hésitations. C'est pour ces pièces, porcelaines et tapisseries notamment, qui sont, par leur qualité et leur préciosité, «à la limite » des critères d'entrée au Muséum mais qui finalement ne l'intègrent pas, que l'on adopte cette forme de sélection intermédiaire que nous évoquions, consistant à les faire momentanément échapper à l'aliénation. C'est le cas à Versailles de dizaines de tapis d'Aubusson et de Savonnerie - à la demande de la commission temporaire des arts. ${ }^{16}$ À Marly, ce sont treize vases «fort riches » en porcelaine de Sèvres qui, ignorés dans les sélections des commissions artistiques, sont distraits pour leur valeur à l'initiative des organisateurs de la vente. ${ }^{17}$ "Quoique non réservés par Boizot [ils] pourraient, écriventils au Conservatoire des arts le 6 pluviôse an II, figurer au Muséum. » Ils ne sont finalement pas, malgré leur beauté, « jugés dignes d'être portés au Muséum »"

Quelques conclusions générales peuvent être dégagées de ces faits distincts car, au-delà des jugements de valeur portés sur les pièces mobilières intégrées au Muséum au moment de leur sélection (moderne, belle, précieuse, etc.), des tendances générales semblent à l'œuvre. La préciosité n'est pas le premier critère de sélection, mais la singularité. Est conservé ce qui sort de l'ordinaire, ce qui est « curieux » (le mot revient souvent) et donc rare. Dans les tables en pierre dure que l'on sélectionne, c'est avant tout la pierre que l'on regarde, sa longueur souvent exceptionnelle, le caractère unique des veines de son marbre. C'est peut-être, au-delà du meuble, le "morceau d'histoire naturelle ${ }^{19}$ » qu'il représente, que l'on admire. C'est ensuite le tour de force artisanal qui motive la sélection, entraîne la soustraction systématique des productions de Boulle et fait regretter à Mathieu la vente des tables de bois pétrifié - où le "travail d'art " avait « embelli la nature ». Bref, ce sont tous les meubles qui, de l'avis des révolutionnaires, échappent au sériel, au multiple - alors que la majorité était issue de commandes en série ${ }^{20}$. Cette donnée renvoie en fait au caractère nécessairement sélectif des procédures révolutionnaires de tri, que l'on applique d'ailleurs aussi bien dans le champ des arts décoratifs que dans celui des beaux-arts. La distraction des ventes des «monuments des arts » ne doit pas seulement répondre à des impératifs typologiques (qui conduiraient, par exemple, à soustraire des ventes tous les tableaux). Au contraire, sculptures et peintures font l'objet de sélections critiques : celles de qualité médiocre, mais aussi les doubles et les œuvres redondantes suivent ainsi le chemin de l'aliénation. C'est la raison pour laquelle des peintures furent dispersées lors des ventes du mobilier de Versailles, Marly, Saint-Cloud et Bellevue. Le 17 septembre 1793 d'ailleurs, le ministre de l'Intérieur Jules-François Paré écrivait à la commission des monuments pour contester officiellement les distractions envisagées par ses commissaires lors de leurs missions à Marly, Rambouillet et Saint-Germain, les objets ne paraissant pas « d'une assez grande 
importance » pour rejoindre les collections du Muséum de Paris. En somme, le ministre regrettait que la Commission n'ait pas été suffisamment «sélective», en particulier à l'égard de peintures de Natoire et de Vouet ${ }^{21}$. Ce refus de l'accumulation apparaît bientôt comme la donnée essentielle des réflexions sur la constitution des collections nationales. Dans un projet d'instruction anonyme émanant de la Commission des monuments sur l'installation de musées dans les églises nationalisées et qui évoque l'élaboration de leurs fonds, on prévoit ceci: "Quant aux objets qui sont communs et qui se trouveront en beaucoup plus grand nombre que les musées, il suffira à chaque musée d'en posséder deux, le reste sera vendu ${ }^{22}$.»

8 Ces réflexions ont bien entendu des conséquences sur la sélection des meubles. Il semblerait ainsi qu'à Versailles, un meuble de Riesener, le secrétaire du comte de Provence (ill. 2) «allait être retiré de la vente comme pièce d'art précieuse pour être conservé au Muséum, d'après le décret relatif à ces objets ; mais y ayant été trouvés deux à peu près semblables dans le Garde-meuble, on s'est borné à n'en conserver qu'un ${ }^{23}$ ».

\section{2: Jean-Henri Riesener, Secrétaire à cylindre du comte de Provence à Versailles}

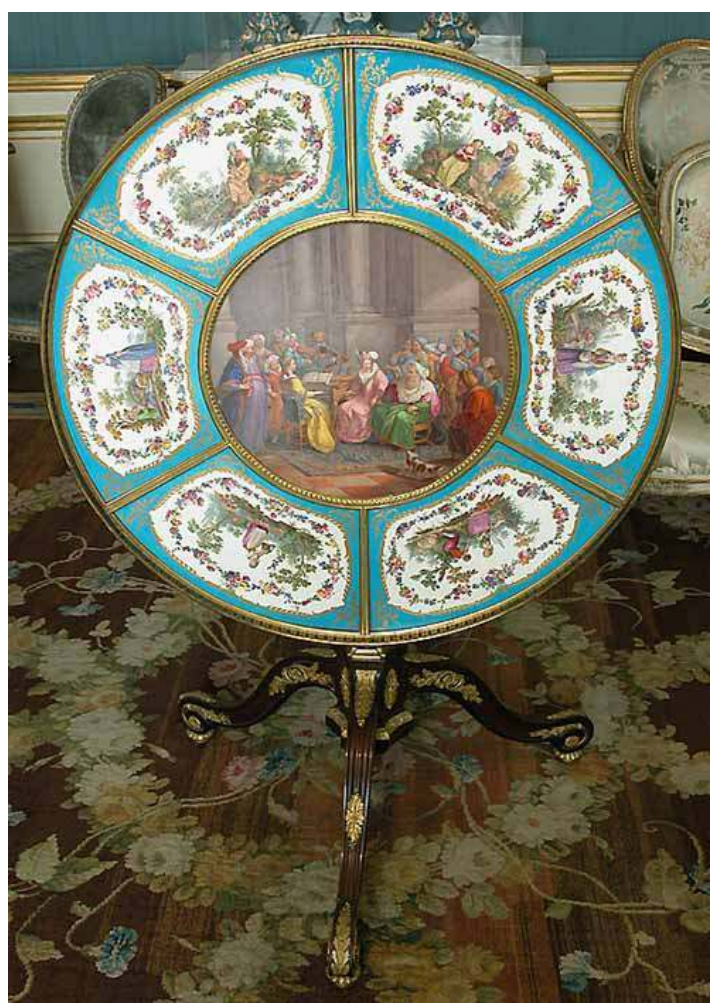

1774, Waddesdon Manor

CL. NATIONAL TRUST, WADDESDON MANOR

9 Une idée de l'aboutissement de ces considérations sur la place du multiple dans le musée de la République est enfin donnée dans l'exemple significatif du guéridon de la comtesse du Barry à Louveciennes (ill. 3). 
III. 3: Charles-Nicolas Dodin et Martin Carlin, Table dite " guéridon de madame du Barry "

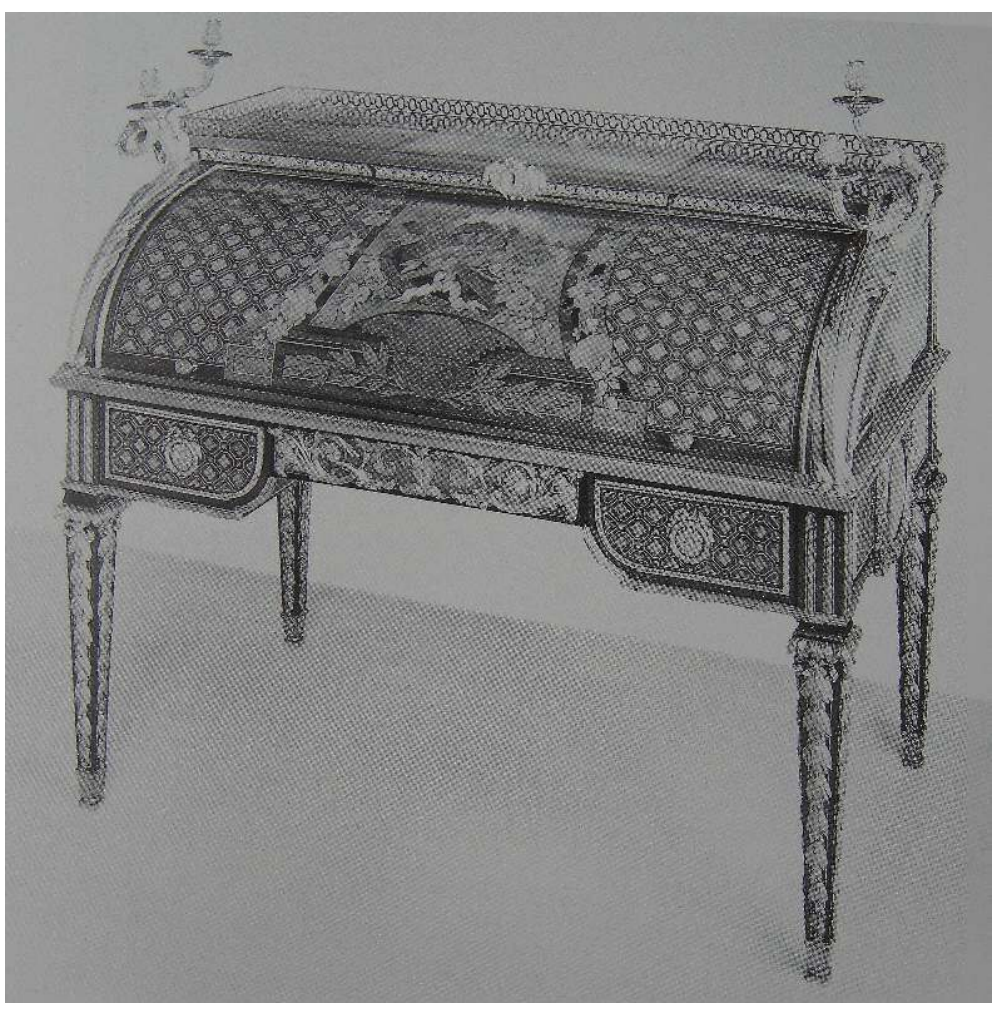

1774, musée du Louvre

Cl. Musée du Louvre/A. Dequier.

Ce meuble d'exception, dont le plateau était orné de plaques en porcelaine tendre de Sèvres, fait sans surprise partie des objets sélectionnés sur décision de la commission des arts locale, en pluviôse an II. Pourtant, des voix discordantes s'élèvent. La commission des subsistances, qui a alors la charge d'écouler selon de nouvelles modalités certaines parties du mobilier nationalisé, milite pour son aliénation. Au printemps 1794, elle dresse à Louveciennes :

[L']état des objets enlevés chez la Dubary [sic] par la Commission des arts de Versailles, et jugés n'être pas faits pour ouvrir un Muséum d'autant que la République possède et possèdera toujours des ouvriers et des fabricants en état de reproduire les mêmes objets ${ }^{24}$.

L'argument avancé ne relevait pas uniquement de l'ordre de l'intérêt financier et donne un ultime éclairage sur la position des meubles à l'heure de la sélection "patrimoniale » car si la singularité de certains ne faisait aucun doute, leur plus grand défaut était d'être non seulement multiples, mais duplicables. 


\section{NOTES}

1. Exposé succinct des travaux de la Commission des monuments depuis son établissement en novembre 1790, 3 frimaire II.

2. Je souligne.

3. Rapport fait à la Convention au nom du Comité d'instruction publique, par Mathieu, député, le 28 frimaire, l'an $2 e$ de la République française. Le rapport entier est édité dans Jean Guillaume éd., Procès-verbaux du comité d'Instruction publique de la Convention nationale, Paris, 1897, vol. III, p. 171-181.

4. La question a été soulevée par Marianne Clerc dans un article publié dans les Cahiers du CRHIPA [Centre de recherche en histoire et histoire de l'art. Italie, pays alpins] de l'université de Grenoble. "Le mobilier à l'heure du choix révolutionnaire: la notion d'œuvre d'art en question? », Cahiers n 3, 2000, « Musées perdus, musées retrouvés », p. 91-99.

5. Jean-Baptiste Duvergier, Collection complète des lois, vol. V, Paris, 1825. « Décret portant réunion des commissions établies pour la conservation des monuments des arts et des sciences ", 18 octobre 1792, p. 30-31.

6. Voir la fameuse «Instruction concernant la conservation des monuments » du 15 décembre 1790.

7. Louis Tuetey éd., Procès-verbaux de la Commission des monuments, pièce annexe XXIX, Paris, 1902, vol. I, p. 324-325.

8. Louis Tuetey éd., Procès-verbaux de la Commission des monuments, pièce annexe XVII, Paris, 1902, vol. I, p. 305-306.

9. Louis Courajod, Alexandre Lenoir. Son journal et le musée des monuments français, Paris, H. Champion, 1878, vol. I, p. 122.

10. Lettre des commissaires du conseil exécutif provisoire et du district de Versailles «aux membres du Conservatoire des arts ", 6 pluviôse an II. Arch. dép. Yvelines, 2Q73, pièce $n^{\circ} 29$.

11. Louis Tuetey éd., Procès-verbaux de la Commission des monuments, op. cit., p. 314-315.

12. Ibid., p. 314-315.

13. "Inventaire des objets sélectionnés par la Commission des monuments », 11 frimaire an II. Arch. dép. Yvelines, 2 Q73.

14. Marianne Clerc, « Le mobilier à l'heure du choix révolutionnaire : la notion d'œuvre d'art en question?», op. cit., p. 93-94.

15. «Inventaire des objets sélectionnés par la Commission des monuments », 11 frimaire an II. Arch. dép. Yvelines, 2 Q73.

16. «Procès-verbal d'inventaire des meubles [...] qui n'ont pu être vendus à la vente du mobilier de la ci-devant liste civile à Versailles », 22 fructidor an II. Arch. dép. Yvelines, 2 Q66.

17. L'implication des autorités administratives dans les politiques de sélection fut très forte lors de l'organisation des ventes du mobilier des résidences royales.

18. Lettre des commissaires du conseil exécutif provisoire et du district de Versailles «aux membres du Conservatoire des arts ", 6 pluviôse an II. Arch. dép. Yvelines, 2Q73, pièce $n^{\circ} 29$.

19. C'est l'expression qu'utilise Mathieu dans son rapport, à propos des tables de MarieAntoinette.

20. Daniel Meyer, «Les meubles sont-ils vraiment des œuvres d'art? ", Connaissance des arts, $\mathrm{n}^{\circ}$ 433, mars 1988, p. 86-97.

21. Louis Tuetey éd., Procès-verbaux de la Commission des monuments, op. cit., vol. II, p. 13.

22. Ibid., vol. I, p. 256-267. 
23. C'est ce que précise une curieuse liste, publiée en Hollande en 1794, de meubles vendus à Versailles. Charles Davillier, «La vente du mobilier de Versailles pendant la Terreur », Gazette des Beaux-arts, Paris, 1877, 30 p.

24. Stéphane Grandjean, «Le guéridon de Madame du Barry provenant de Louveciennes », Revue du Louvre, $\mathrm{n}^{\circ} 1$, Paris, 1979, p. 44-49.

\section{RÉSUMÉS}

La Révolution française est, d'un point de vue patrimonial, le moment du choix. Les vagues de nationalisation successives viennent gonfler la masse du mobilier destiné à l'aliénation et, en contrepoint, donnent aux révolutionnaires l'occasion de « patrimonialiser » (nous entendons, par patrimonialiser, le fait d'intégrer après sélection un certain nombre des effets confisqués à un corpus rendu inaliénable: celui du patrimoine de la nation) un certain nombre de "monuments » finalement conservés. L'exercice de la vente, marquant ainsi la genèse du "geste de choisir ", s'est donc accompagné de celui du tri, de la distraction, d'autant plus délicat à saisir que les contours en sont toujours restés flous et que ses critères n'ont jamais été clairement définis. Audelà donc du caractère nécessairement arbitraire de ces sélections ("Les œuvres d'art avaient leur tribunal révolutionnaire », disait l'historien d'art Louis Courajod), des tendances générales ont été à l'œuvre que les soustractions opérées lors des ventes des meubles des résidences royales, organisées entre 1793 et 1795, permettent de restituer. Elles relativisent l'idée de distractions uniquement typologiques et mettent en exergue le principe implicite qui y a présidé: ne garder que l'unique, que ce qui échappe au sériel - même dans le champ des beaux-arts. Face à la richesse quantitative de ce mobilier, les distractions patrimoniales révolutionnaires se font inévitablement sélectives; le refus de l'accumulation apparaît alors comme la donnée essentielle des réflexions sur la constitution des collections nationales. On comprend aisément, à cet égard, le problème que posaient les meubles meublants, même d'exception: si les commissions préposées aux distractions affirmaient explicitement qu'ils avaient bien leur place dans les sélections, la règle tacite qu'elles appliquaient condamnait majoritairement ces productions, issues de commandes en série, à l'aliénation. Des objets ont pourtant suscité, au cours du processus de sélection, questionnements et hésitations. Une solution intermédiaire put alors être adoptée, ce qui ne justifiait pas totalement une distraction patrimoniale, qui fit néanmoins l'objet d'une sélection "commerciale » soustrayant temporairement le meuble à la vente. Les cas des tables en pétrification de Marie-Antoinette ou du guéridon de la du Barry se ressentent de ces inflexions et donnent un ultime éclairage sur la position des meubles à l'heure de la sélection "patrimoniale», car si la singularité de certains ne faisait aucun doute, leur plus grand défaut était d'être non seulement multiples, mais « duplicables ».

French revolution was, in the history of the creation of public collections, the moment of choice. While some of the furniture and works of art which had been nationalized were intended to be auctioned, others were regarded as « monuments" and preserved. The criteria for that selection were never clearly worked out, and that is why it can often appear as arbitrary (« Works of art had their Revolutionary tribunal ", said art historian Louis Courajod). But a general pattern can nevertheless be described while looking at the selections which were done before royal furniture was auctioned, between 1793 and 1795. Thus, selections were not only done according to typological criteria, but following a general principle: keeping what was regarded as unique, 
what had not been serially produced. From this point of view, the very first demand of the revolutionary authorities was to be selective, and the issue raised by the furniture is easily understood - since they rarely were unrepeatable. Marie-Antoinette's petrified wood tables and Du Barry's pedestal table cases throw light on that difficulty, and on the ambiguous position occupied by the furniture in the selections.

Was das Nationalerbe anbelangt, erweist sich die französische Revolution als die Zeit der Auslese. Die aufeinanderfolgenden Nationalisierungswellen wurden zum Anlass, eine beträchtliche Menge von Mobiliar, das enteignet werden sollte, zu sammeln. Es gab für die Revolutionäre Anlass zu „patrimonialiser“, d.h. konfiszierte Besitzgüter nach Selektion als „Kunstdenkmal“ in die unübertragbar gewordene Korpusliste des Nationalerbes einzutragen. Durch die praktische Erfahrung der Verkäufe entwickelte sich die „Geste der Selektion“, deren ungenaue Umrisse und nie festgesetzte Kriterien schwer aufzufassen sind. „ Auch die Kunstwerke hatten ihr Revolutionstribunal “, schrieb der Kunsthistoriker Louis Courajod. Über die unvermeidliche Willkürlichkeit des Auswählens hinaus führt die Beobachtung der Verkäufe des Mobiliars der königlichen Wohnstätten zwischen 1793 und 1795 einige allgemeine Gesichtspunkte vor Augen. Es wurden nicht nur typologische Kriterien herangezogen, sondern auch dieses stillschweigende Prinzip, dass nur Einzelstücke zu behalten seien, was jede Serienanfertigung ausschloss. So geschah es auch im Bereich der bildenden Künste. Angesichts der Anzahl der Angebote trafen also die Revolutionäre eine selektive Auswahl für das Kulturerbe. Das wesentliche Kriterium in der Erstellung der nationalen Kunstsammlungen scheint die Abneigung gegen Anhäufung zu sein. Aus diesem Grund wurden alle Möbel, die vornehmeren inbegriffen, in Frage gestellt : selbst wenn die Kommissionsmitglieder ihnen einen wertvollen Platz in der Auslese ausdrücklich anerkannten, hielten sie sich doch an die stillschweigende Regel, mehrfach bestellte Möbel auszuschließen. Im Lauf des Auswahlvorgangs gaben gewisse Stücke sogar Anlass zu schwierigen Abwägungen, die manchmal $\mathrm{zu}$ Kompromissen führten. Was sich dem Nationalerbe beispielsweise als nicht direkt geeignet erwies, konnte doch aus kommerziellen Gründen dem Verkauf momentan entzogen werden. Besonders repräsentativ ist der Fall der Tische aus versteinertem Holz von Marie-Antoinette oder der des Leuchtertisches der du Barry. Bei dieser Auswahl für das „Nationalerbe“ war selbstverständlich der einzigartige künstlerische Wert gewisser Angebote unbestritten, aber als deren hauptsächliche Schwäche galt nicht nur ihre Anzahl sondern ihr „duplizierbares“ Gepräge.

\section{AUTEUR}

\section{RÉMI GAILLARD}

Rémi Gaillard, archiviste paléographe, a soutenu en juin 2010 un master 2 intitulé « Un encan pur et simple »? Un aperçu de l'organisation des ventes révolutionnaires du mobilier royal (1793-1795). Il a soutenu en mars 2011 sa thèse d'établissement, dir. de Jean-Michel Leniaud, intitulée « Après qu'il s'est assemblé marchands-fripiers, tapissiers, revendeurs et autres citoyens ». Étude institutionnelle des ventes révolutionnaires du mobilier royal, 1793-1795 (Versailles, Marly, Saint-Cloud, Bellevue), pour laquelle il a obtenu le prix Auguste-Molinier qui récompense la meilleure thèse de la promotion. 